\title{
3D Segmentation of Brain Tumor Imaging
}

\author{
M. Sumithra ${ }^{1}$, P. Madhumitha ${ }^{2}$, S. Madhumitha ${ }^{3}$, D. Malini ${ }^{5}$, B. Poorni \\ Vinayaa $^{5}$
}

\author{
${ }^{1}$ Associate Professor, Department of Information Technology, Panimalar Engineering College, Chennai, India \\ 2,3,4,5 Department of Information Technology, Panimalar Engineering College, Chennai, India
}

\begin{abstract}
A brain tumor is a collection of anomalous cells that grow in or around the brain. Brain tumors affect the humans badly, it can disrupt proper brain function and be life-threatening. In this project, we have proposed a system to detect, segment, and classify the tumors present in the brain. Once the brain tumor is identified at the very beginning, proper treatments can be done and it may be cured.
\end{abstract}

Keywords - tumor, life-threatening, cancer.

\section{INTRODUCTION}

A tumor is a collection or mass of abnormal cells that occur in various parts of the body. A tumor can result in cancer, which is the main reason for death and accounts for around $13 \%$ of every death world-wide. The cancer occurrence rate is rising at an alarming rate in the world. Therefore, tumor detection is significant in previous stages. The mast of abnormal cells that grow in or around the brain is called a brain tumor. It poses a risk to the healthy brain by either destroying or invading normal brain tissue. Brain tumors can be malignant (brain cancer) or benign (do not contain cancer cells). They may be primary (originate in the brain) or metastatic (cancer cells elsewhere in the body travel to the brain). The exact cause of brain cancer is unknown. However, factors that can elevate the risk of brain cancer include exposure to high doses of ionizing radiation and a family history of brain cancer. If brain cancer had occurred the doctor may order for imaging tests like MRI, head CT, and head PET. But MRI is the most effective and extensively used technique for brain tumor detection. Treatment will depend on the size and type of the tumor, its growth rate, and the person's general health. Options include radiation therapy, chemotherapy, surgery, targeted biological therapy, or a combination thereof. Brain tumors are categorized based on where the tumor is located, the type of tissue involved, whether the tumor is benign or malignant, and other factors.

Identification of brain tumor from MRI consist of different stages. Segmentation is termed to be a significant but tough step for the classification of medical imaging and its analysis. Therefore, it is important that the segmentation of the MRI images should be done precisely before asking the processor for an accurate diagnosis. This project has focused on verifying the accuracy and efficiency of brain tumor detection via segmentation technique for MRI images of the brain.

\section{LITERATURE SURVEY}

\section{Brain Tumor Segmentation Using Convolutional Neural Network In MRI}

Brain tumor segmentation methodology is based on Convolutional Neural Networks ( $\mathrm{CNN})$, by exploring into small $3 \times 3$ kernels. The employment of small kernels permits coming up with a deeper architecture, besides having a positive impact against overfitting, given the less variety of masses within the network and also investigating on the utilization of intensity normalization as a preprocessing step, which is not common in Convolution Neural Network-based segmentation methods, and welltried in conjunction with information augmentation to be intolerably in effect for neoplasm segmentation in magnetic resonance images.

2. Semantic Image Segmentation with Deep Convolutional Nets, Atrous Convolution, and Fully Connected CRFS

In this paper, the task of semantic image segmentation with Deep Learning is labeled and made some main contributions that are experimentally shown to have substantial merit. First, it highlights convolution with upsampled filters, or 'atrous convolution', as a powerful tool in dense prediction tasks. Atrous convolution allows us to directly control the resolution at which feature responses are computed within Deep Convolutional Neural Networks. It also allows us to successfully enlarge the field of view of filters to incorporate larger context without elevating the 
number of parameters or the amount of computation. Second, atrous spatial pyramid pooling (ASPP) offers to robustly segment objects at multiple scales. ASPP inspects an incoming convolutional layer with filters at multiple sampling rates and constructive fields-of-views, thus acquiring image context at multiple scales.

we also improve the localization of object boundaries by integrating methods from DCNNs and probabilistic graphical models.

\section{The Multimodal Brain Tumor Image Segmentation Benchmark (BRATS)}

In this paper, it reports the structure and consequence of the Multimodal Brain Tumor Image Segmentation Benchmark organized in conjunction with the MICCAI 2012 conference. 20 advanced tumor segmentation algorithms were put into a set of 65 multi-contrast MRI (Magnetic Resonance Imaging) scans of low-grade and high-grade glioma patients manually interpreted and to 65 comparable scans generated using tumor image simulation program. Quantitative evaluations revealed a substantial dispute between the human raters in segmenting various tumor sub-regions illustrating the difficulty of the task. It is found that different algorithms worked best for different sub-regions (reaching performance comparable to human variability), but that no single method ranked in the top for all sub-regions simultaneously.

\section{Brain Tumor Segmentation by a Generative Model with a Prior on Tumor Shape}

This paper presents a fully automated generative method for brain tumor segmentation in multi-modal magnetic resonance images. It bases the method on the type of generative model often used for healthy brain tissues, where tissues are modeled by Gaussian mixture models combined with a spatial tissue prior. We extend the basic model with a tumor prior, which uses convolutional restricted Boltzmann machines to the model tumor shape. Experiments on the 2015 and 2013 BRATS data sets indicate that the method's performance is comparable to the current state of the art in the field while being readily extendable to any number of input contrasts and not tied to any specific imaging protocol.

\section{NiftyNet: a deep-learning platform for medical imaging}

This paper presents the open-source NiftyNet platform for deep learning in medical imaging. The NiftyNet infrastructure enables a deep-learning pipeline for a large scale of medical imaging applications together with segmentation, image generation, regression, and representation learning applications. Components of the NiftyNet pipeline including data loading, network architectures, data augmentation, loss functions, and evaluation metrics. NiftyNet is built on the TensorFlow framework and supports certain features like TensorBoard visualization and computational graphs by default. The NiftyNet infrastructure helps researchers to swiftly develop deep learning solutions for regression, segmentation, image generation, and representation learning applications.

\section{EXISTING SYSTEM}

The existing system describes the cellular automation of segmentation. The technique is used in interactive multilabel segmentation for $\mathrm{N}$-dimensional images. It segments the areas which are more difficult to segment. The method is iterative, giving feedback to the user while the segmentation is computed.

\section{PROPOSED SYSTEM}

In this project, MRI Brain images of a patient are taken as input. The images are pre-processed and further segmented for the required feature. Then feature Extraction is done for the images by GLCM features. Region of interest (ROI) segmentation is applied in order to identify the affected portion of tumor. Here the threshold required for segmenting adjusts itself according to the segmented area and position. Finally, classification applied through deep neural networks then results in the image that will be compared with the dataset images and it will display whether it is benign or malignant.

\section{MODULES}

1.

\section{PREPROCESSING}

2.

\section{SEGMENTATION}

\section{BACK PROBAGATIO N}

\section{PREPROCESSING}

In this module, if the input images are color images they are converted to grayscale from that color images. In image enhancement, the goal is to highlight certain image features for upcoming analysis or for image display. Some examples are contrast and edge enhancement, noise filtering, sharpening, pseudo-coloring, and magnifying. Image enhancement is useful in feature extraction, image display, and image analysis. The median filter is used in order to remove the noise from the image. Median filtering is a nonlinear operation frequently used in image 
processing to diminish salt and pepper noise. A median filter is more effective than convolution when the goal is to simultaneously decrease noise and preserve edges. Here the region of Interest (ROI) algorithm is used in order to segment the portion of defected areas.

\section{SEGMENTATION}

In this module, image features like color, weight, depth, and pixel information are to be applied before the classification. Image segmentation is usually used to locate objects and boundaries in images. Explicitly image segmentation is the process of allocating a label to every pixel in an image such that pixels with the same label share certain characteristics. The outcome of image segmentation is a set of segments that collectively cover the entire image or a set of outline extracted from the image. Each of the pixels in a stretch is alike with respect to some characteristic or computed property, such as color, intensity, or texture.

\section{BACK PROBAGATION}

This module is used to establish the backpropagation neural network concept for training the image and testing the image with the help of a weight estimating classifier. The segmented image is made to undergo backpropagation. In backpropagation, the segmented image is compared with the dataset images. During the comparison, the segmented tumor is checked for resemblance that may be present within the dataset. After comparison, it will display whether the tumor is normal or abnormal.

\section{SCREENSHOTS}

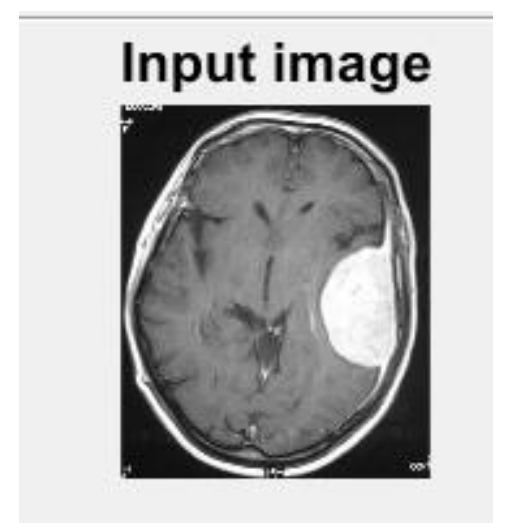

Fig.1: Input image

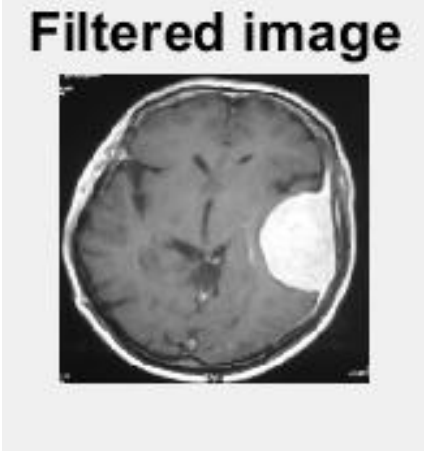

Fig.2: Filtered image

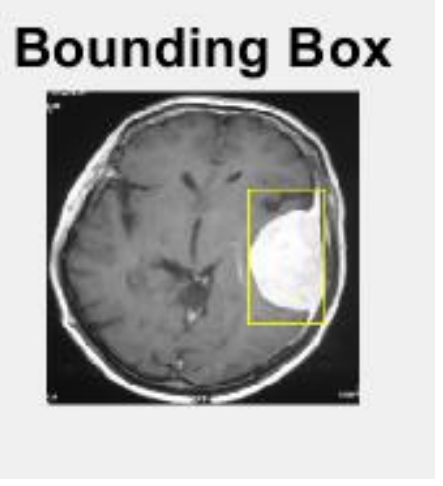

Fig.3: Bounding image

\section{tumor alone}

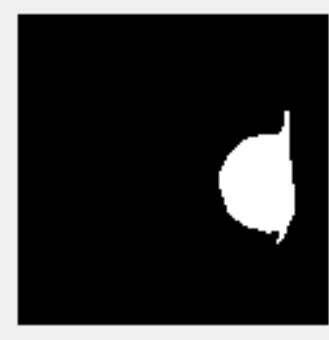

Fig.4: Tumor image

\section{Tumor Outline}

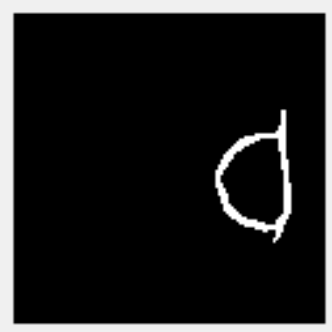


Fig.5: Outline image

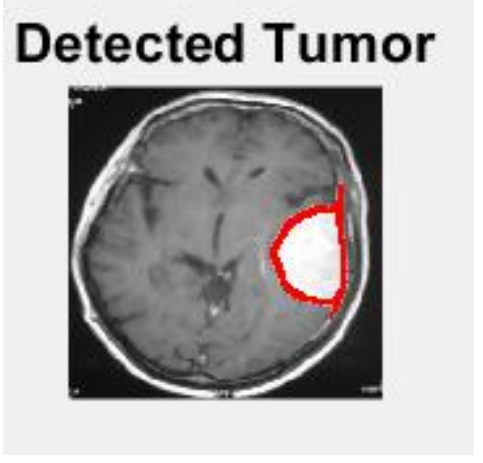

Fig.6: Final image

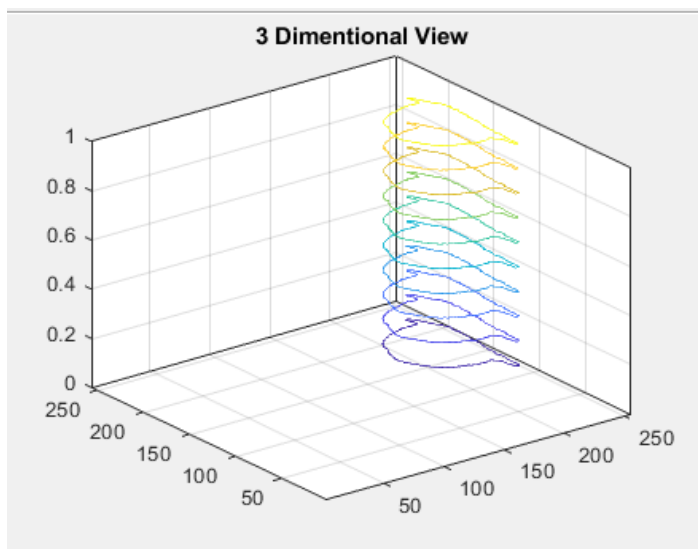

Fig.7: 3 Dimensional image

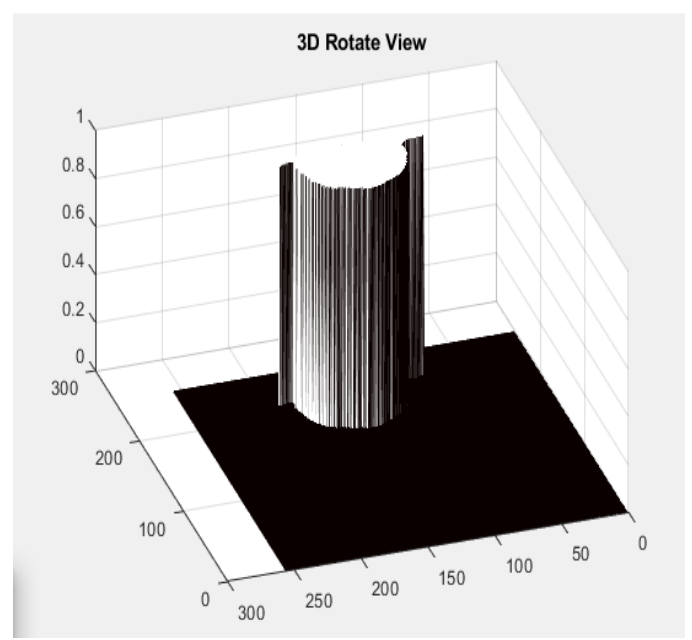

Fig.8: 3 Dimensional rotate view

\section{CONCLUSION}

Anomalous growth of tissues in the brain which affect proper brain functions is considered as a brain tumor. The main goal of medical image processing is to identify meaningful and accurate information using images with the least error possible. Brain tumor identifications through MRI (Magnetic Resonance Imaging) images is a tedious task because of the complexity of the brain. These tumors can be efficiently segmented using various image segmentation techniques. The process of identifying brain tumors through MRI images can be classified under four different sections; pre-processing, image segmentation, feature extraction, and image classification. Thresholding is an effective and easiest approach among image segmentation techniques. It easy to implement and frequently used these days. When the contrast between the foreground object and the background object is comparatively high, the threshold technique works well. Finally, the message box will display whether the tumor is normal or abnormal.

\section{FUTURE ENHANCEMENT}

Future work would utilize a more sophisticated segmentation algorithm. Upon successful implementation of the proposed system, another system would be developed to detect, segment, and classify tumor cells based on the MRI image with a very high level of accuracy.

\section{REFERENCES}

[1] B. H. Menze et al., "The multimodal brain tumor image segmentation benchmark (brats)," IEEE Trans. Med. Imag. vol. 34, no. 10, pp. 1993-2024, Oct. 2015.

[2] M. I. Razzak and S. Naz, "Microscopic blood smear segmentation and classification using deep contour aware cnn and extreme machine learn- ing," in Proc. IEEE Conf. Comput. Vis. Pattern Recognit. Workshops, 2017, pp. 801807.

[3] M. I. Razzak and B. Alhaqbani, "Automatic detection of malarial parasite using microscopic blood images," J. Med. Imag. Health Informat., vol. 5, no. 3, pp. 591-598, 2015.

[4] M. Havaei et al., "Brain tumor segmentation with deep neural networks," Med. Image Anal., vol. 35, pp. 18-31, 2017.

[5] M. Winkels and T. S. Cohen, "3D g-CNNs for pulmonary nodule detec- tion," Int. Conf. Med. Imag. Deep Learn. (MIDL), 2018.

[6] S. Pereira, A. Pinto, V. Alves, and C. A. Silva, "Brain tumor segmentation using convolutional neural networks in MRI images," IEEE Trans. Med. Imag., vol. 35, no. 5, pp. 12401251, May 2016.

[7] Zhao, Y.Wu, G. Song, Z. Li, Y.Zhang, and Y.Fan, “A deep learning model integrating FCNNs and CRFs for brain tumor segmentation," Med. Image Anal., vol. 43, pp. 98111, 2018.

[8] K. Kamnitsas et al., "Efficient multi-scale 3D CNN with 
fully connected CRF for accurate brain lesion segmentation," Med. Image Anal., vol. 36, pp. 61-78, 2017.

[9] R. Saha, A. Phophalia, and S. K. Mitra, "Brain tumor segmentation from multimodal $\mathrm{mr}$ images using rough sets," in Proc. Int. Conf. Comput. Vision, Graph., Image Process., 2016, pp. 133-144.

[10] P. D. Chang, "Fully convolutional neural networks with hyperlocal features for brain tumor segmentation," in Proc. MICCAI-BRATS Workshop, 2016, pp. 4-9.

[11] T. Lun and W. Hsu, "Brain tumor segmentation using deep convolutional neural network," in Proc. BRATS-MICCAI, 2016, pp. 1240-1251.

[12] Casamitjana, S. Puch, A. Aduriz, E. Sayrol, and V. Vilaplana, "3D convolutional networks for brain tumor segmentation," in Proc. MIC-CAI Challenge Multimodal Brain Tumor Image Segmentation, 2016, pp. 65-68.

[13] P. Dvorak and B. Menze, "Structured prediction with convolutional neural networks for multimodal brain tumor segmentation," in Proc. Multimodal Brain Tumor Image Segmentation Challenge, 2015, pp. 13-24.

[14] Zhao, Y. Wu, G. Song, Z. Li, Y. Fan, and Y.Zhang, "Brain tumor segmentation using a fully convolutional neural network with conditional random fields," in Proc. Int. Workshop Brainlesion: Glioma, Multiple Sclerosis, Stroke Traumatic Brain Injuries, 2016, pp. 75-87.

[15] V. S. Chagas et al., "3D u-nets for brain tumor segmentation in MICCAI 2017 brats challenge," in Proc. Int. MICCAI BraTS Challenge, 2017, pp. 9-14.

[16] M. Sumithra and S. Malathi, "A survey on Medical Image Segmentation Methods with Different Modalitites", International Journal of Engineering Research and Technology (IJERT) - Volume 6 Issue 2, Mar 2018.(gs-2).

[17] M. Sumithra and S. Malathi, "A Survey of Brain Tumor Segmentation Methods with Different Image Modalitites", International Journal of Computer Science Trends and Technology (IJCST) - Volume 5 Issue 2, Mar - Apr 2017.(gs-3) 